\title{
PulseNet Lebanon: An Overview of Its Activities, Outbreak Investigations, and Challenges
}

\author{
Sukayna M. Fadlallah, ${ }^{1}$ Marwa Shehab, ${ }^{1}$ Katia Cheaito,, Nathaline Haidar-Ahmad, ${ }^{1}$ Bassam El Hafi, \\ Majd Saleh, ${ }^{2}$ Zeina Nasser, ${ }^{2}$ Rima El Hajj, ${ }^{3}$ Nada Ghosn, ${ }^{2}$ Walid Ammar, ${ }^{2}$ and Ghassan M. Matar ${ }^{1}$
}

\begin{abstract}
Background: Foodborne diseases are still a major health issue in Lebanon, although some steps have been taken forward in food safety. To this purpose, PulseNet Lebanon, a foodborne diseases tracking network, was established in 2009, through the collaboration between the Ministry of Public Health (MoPH) and the American University of Beirut (AUB).

Materials and Methods: Three papers published regarding the PulseNet project were summarized. Initially, clinical and food samples, collected within the surveillance network scope, were identified by using the respective API for Salmonella and Listeria spp. Salmonella spp. were further serotyped by using the Kauffman and White method. Campylobacter spp. were determined by the $16 \mathrm{~S}$ rRNA sequencing method. Antimicrobial susceptibility to a number of antibiotics was determined by using the disk diffusion method for Samonella and Campylobacter spp. Genomic diversity was determined by using pulsed field gel electrophoresis (PFGE) and random amplified polymorphic DNA (RAPD). Results: Results indicated that 290 clinical and 49 food isolates were identified as Salmonella. Serotyping revealed the prevalence of ten and seven serotypes in the clinical and food samples, respectively. Fifty-one isolates from chicken ceca and carcass were identified to be Campylobacter spp. Fifty-nine samples were identified to be Listeria monocytogenes. Antimicrobial susceptibility testing revealed a wide range of resistance among the different samples. PFGE showed a variation in pulsotypes among the Salmonella serotypes. PFGE also linked certain outbreaks to their food sources. This method also demonstrated 13 subtypes with $100 \%$ similarity among the L. monocytogenes isolates. Finally, the Camplyobcater spp. were grouped into nine clusters with a minimum similarity of $43.5 \%$ using RAPD.

Conclusion: This summary of results shows the importance of implementing a "farm-to-fork" approach in the surveillance of foodborne disease outbreaks in Lebanon, allowing the detection of pathogens causing foodborne disease outbreaks in a timely fashion.
\end{abstract}

Keywords: PulseNet, foodborne, disease, Salmonella, Campylobacter, Listeria, outbreak

\section{Introduction}

D ESPITE ADVANCEMENTS IN TECHNOLOGY, foodborne diseases remain a global issue that results in health and economic burdens. Sixty-six percent of reported foodborne illnesses are caused by bacteria (Addis and Sisay, 2015). These bacteria include: Salmonella spp., Escherichia coli, Listeria monocytogenes, Campylobacter spp., Clostridium perfringens, and others (Wolfram, 2017). In 2010, 503 cases of foodborne diseases in Lebanon were identified by clinical laboratories and clustered into 42 episodes of foodborne disease outbreaks, of which Salmonella spp. was the most common causative agent. In 2011, the epidemiological surveillance unit at the Ministry of Public Health (MoPH) reported 311 cases of food poisoning and 362 cases of typhoid fever that year, even though salmonellosis is caused

\footnotetext{
${ }^{1}$ Department of Experimental Pathology, Immunology and Microbiology and Center for Infectious Diseases Research, Faculty of Medicine, American University of Beirut, Beirut, Lebanon.

${ }^{2}$ Ministry of Public Health, Beirut, Lebanon.

${ }^{3}$ Lebanese Agriculture Research Institute, Fanar, Lebanon.

(C) Sukayna M. Fadlallah et al. 2019; Published by Mary Ann Liebert, Inc. This Open Access article is distributed under the terms of the Creative Commons License (http://creativecommons.org/licenses/by/4.0), which permits unrestricted use, distribution, and reproduction in any medium, provided the original work is properly cited.
} 


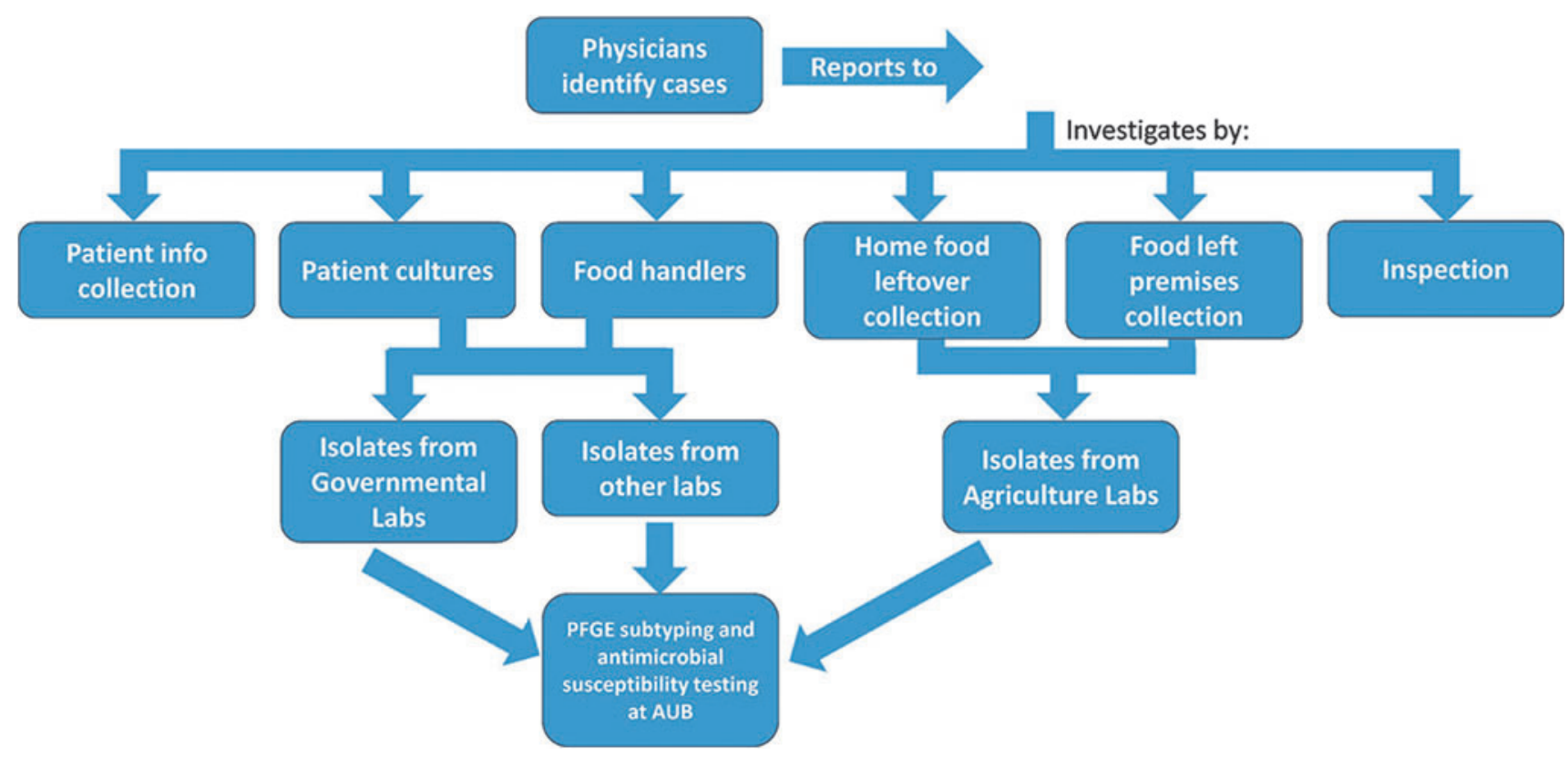

FIG. 1. The laboratory-based surveillance and workflow of PulseNet Lebanon.

by more serotypes than typhoid fever (Unpublished data from MoPH).

In Lebanon, outbreaks are usually detected if spatiotemporal clusters are occurring, or if the exposure history identifies common shared meals (Ghosn et al., 2008). Distributed outbreaks are not detected and are hidden by the endemicity of the disease. Laboratory-based strain surveillance can highly improve surveillance systems of communicable diseases by increasing the specificity of detection (World Health Organization, 2017). Epidemiological surveillance of communicable diseases, including foodborne disease outbreaks, is mandated by law in Lebanon and outbreaks must be reported to the Epidemiological Surveillance Unit at the MoPH in Beirut, Lebanon, on a monthly basis. However, this monthly reporting system delays early detection and data obtained are not useful for timely intervention (Ghosn et al., 2008; World Health Organization, 2012). In addition, undiagnosed cases are common and prevalence data may be biased due to under-reporting. Therefore, the Lebanese chapter of the disease tracking network, PulseNet International, was launched in 2009 in Lebanon between the American University of Beirut (AUB) and the MoPH. This collaboration involves public and private sectors joining forces to reinforce the investigation of foodborne diseases through identifying pathogens causing foodborne disease by time, place, type, subtype, and antimicrobial resistance pattern, and linking clinical cases to their food sources during outbreaks. This will ultimately direct the $\mathrm{MoPH}$ in taking the right preventative measures for foodborne diseases. In addition, the knowledge obtained about the antimicrobial resistance patterns will guide physicians in selecting appropriate treatment regimens.

The main roles of PulseNet Lebanon are to: improve the detection and investigation of foodborne disease (FBD) outbreaks; identify the sources of contamination, vehicles, and dissemination routes; support the recognition and early detection of emerging foodborne pathogens; strengthen the national capabilities for foodborne disease surveillance by facilitating their response capacity, monitoring and investigation of outbreaks; organize and promote training programs and continued education, encouraging the exchange of experience and available resources in the region; promote and strengthen intersectorial participation in the formation and functioning of epidemiology surveillance systems; and exchange information with regional networks of PulseNet International. The workflow of PulseNet Lebanon that links physicians, MoPH, laboratory technicians, and public health workers is shown in Figure 1. PulseNet Lebanon is part of the PulseNet MiddleEast, which promotes communication through yearly meetings that are geared by the steering committee (one of which is PulseNet Lebanon) represented by its members. This collaboration ensures exchange of data and materials under the World Health Organization (WHO) umbrella. In addition, PulseNet Lebanon was selected along with Oman to become the certifier of Salmonella pulsed field gel electrophoresis (PFGE) testing.

\section{Materials and Methods}

Three articles publishing work done on Salmonella spp., Camylobacter spp., and L. monocytogenes from food and clinical isolates were summarized to demonstrate some of the research done since PulseNet establishment in Lebanon. The scope of these studies was to identify the circulating species of the bacterial pathogens, determine the antimicrobial resistance pattern, and link food sources to foodborne illness cases during outbreaks. Regular monitoring consists of collecting food samples such as poultry meat (neck and chicken liver), ceca coming from broiler chicken farms and slaughterhouses, and traditionally consumed Lebanese dairy products from randomly selected manufacturers and outlets to screen for the presence of the pathogenic organisms L. monocytogenes, Salmonella spp., and Campylobacter spp. Hence, all samples in the studies were collected within the scope of constant monitoring of foodborne pathogens and each study had its own set of 
samples isolated within a specific period. In addition, isolates of L. monocytogenes and Salmonella isolated from food submitted routinely to the laboratory of food microbiology at Lebanese Agriculture Research Institute (LARI) during this period were collected and transferred to the reference laboratory at the Faculty of Medicine at the AUB to be stored for further processing.

Clinical samples were obtained from both governmental and private hospitals and these included around 25 hospitals distributed throughout 5 governorates: Beirut, Mount Lebanon, Nabatieh, Bekaa, and the North. Clinical specimens were submitted to both local laboratories and the reference laboratory and were screened for pathogenic isolates.

\section{Identification of isolates}

At the reference laboratory, isolates were identified to the species level by using respective biochemical kits: For Salmonella, API 20E kit (bioMerieux, Marcy L'Etoile, France) and for Listeria, API Listeria kit (bioMérieux) were used (Fadlallah et al., 2016; Haidar et al., 2016). Serotyping for the isolates was performed by latex agglutination using mono- and poly-valent anti-sera for $\mathrm{O}$ and $\mathrm{H}$ antigens according to the Kauffman and White scheme for Salmonella isolates. Sequencing was carried out by using the Big Dye Terminator2.0 kit (Applied Biosystems, CA) according to the manufacturer's instruction. Species identification of the Campylobacter isolates was determined by using the Basic Local Alignment Search Tool (BLAST) (Fadlallah et al., 2018).

\section{Antimicrobial susceptibility testing}

The antimicrobial susceptibility profiles of the isolates were determined by using the disk diffusion agar method following the 2009 CLSI guidelines for Salmonella (Clinical and Laboratory Standards Institute, 2009).

\section{Genotyping}

Genomic relatedness was determined by PFGE on the BioRad CHEF MAPPER (Biorad) using the standard operating procedure for PulseNet PFGE of E. coli O157:H7, E. coli non-O157 (STEC), Salmonella serotypes, Shigella sonnei, and Shigella flexneri with the XbaI (Fermentas, Waltham, MA) as restriction endonuclease (Ribot et al., 2006) and a modified PulseNet protocol for L. monocytogenes (PulseNet International, 2013) with AscI as restriction endonuclease for the Salmonella and Listeria isolates, respectively (Fadlallah et al., 2016; Haidar et al., 2016). Regarding the Campylobacter isolates, random amplified polymorphic DNA (RAPD) analysis was carried out by using the Ready-To-Go RAPD Analysis Beads Kit (GE, Amersham Place, United Kingdom) as per the manufacturer's instructions. All dendrograms were made by using the UPGMA method (unweighted pair group method using arithmetic averages) and Dice similarity coefficient with the BIONUMERICS software (Fadlallah et al., 2018).

\section{Results}

Identification of isolates

Between 2011 and 2014, 290 clinical isolates and 49 food samples were identified to be Salmonella (Fadlallah et al.,
2016). Fifty-nine isolates obtained from local and imported food collected from the Lebanese market were identified to be L. monocytogenes during 2012 and 2013 (Haidar et al., 2016). Also within the same period, 51 samples isolated from chicken ceca samples taken during the evisceration process (38 isolates) and whole poultry carcass (13 isolates) were positive for Campylobacter spp. Samples were identified to be Campylobacter coli by sequencing (Fadlallah et al., 2018). Although $C$. coli is found mainly in pigs, however several studies carried out in Lebanon, Italy, Chile, and Washington showed that this strain is found in chicken carcass and ceca, broiler chicken, turkey breast, and chicken liver respectively (Fernández and Pisón, 1996; Talhouk et al., 1998; Zhao et al., 2001; Pezzotti et al., 2003).

Serotyping indicated that 10 and 7 serotypes were prevalent among the clinical and food isolates respectively. Salmonella Typhimurium, Salmonella Enteritidis, Salmonella Braenderup, Salmonella Typhi, Salmonella Paratyphi A, Salmonella Blockley, and Salmonella Newport were the common serotypes among the clinical and food isolates. The clinical isolates included additional serotypes: Salmonella London, Salmonella Paratyphi B, and Salmonella Paratyphi C. The two most common serotypes in both food and clinical isolates were Salmonella Enteritidis (clinical $=43.4 \%$ and food $=20.4 \%$ ) and Salmonella Typhimurium (clinical, $n=29 \%$ and food, $n=28.5 \%$ ) (Fadlallah et al., 2016). Table 1 shows the distribution of Salmonella serotypes isolated from clinical and food samples.

\section{Antimicrobial susceptibility testing}

Overall, $73.8 \%$ of the clinical samples and $75.5 \%$ of the food samples were susceptible to the four antibiotics tested: ampicillin, trimethoprim-sulfamethoxazole, ciprofloxacin, and ceftazidime in the Salmonella isolates. Regarding the clinical samples, 68 isolates showed resistance to ampicillin, 23 to trimethoprim-sulfamethoxazole, 11 to ciprofloxacin, and 7 to ceftazidime (out of 290 isolates). Among the food strains, 10 isolates showed resistance to ampicillin, 5 to ciprofloxacin, and 1 to ceftazidime (out of 49 isolates). However, they were all susceptible to trimethoprim-sulfamethoxazole (Fadlallah et al., 2016).

Table 1. Distribution of Salmonella Serotypes ISOlated FROM ClinicAl AND FOOD SAMPles (FADlallah ET AL., 2016)

\begin{tabular}{lrc}
\hline & \multicolumn{2}{c}{ Total } \\
\cline { 2 - 3 } Serotype & Clinical & Food \\
\hline Salmonella Typhimurium & $84(29.0)$ & $14(28.5)$ \\
Salmonella Enteritidis & $126(43.4)$ & $10(20.4)$ \\
Salmonella Braenderup & $21(7.2)$ & $4(8.2)$ \\
Salmonella Typhi & $19(6.6)$ & $3(6.1)$ \\
Salmonella London & $10(3.4)$ & - \\
Salmonella Paratyphi A & $8(2.8)$ & $8(16.3)$ \\
Salmonella Blockley & $3(1.0)$ & $2(4.1)$ \\
Salmonella Paratyphi B & $2(0.7)$ & $\overline{(8.2)}$ \\
Salmonella Newport & $2(0.7)$ & $\overline{(8.2)}$ \\
Salmonella Paratyhi C & $2(0.7)$ & $49(100)$ \\
Other & $13(4.5)$ & \\
Total & $290(100)$ & \\
\hline
\end{tabular}



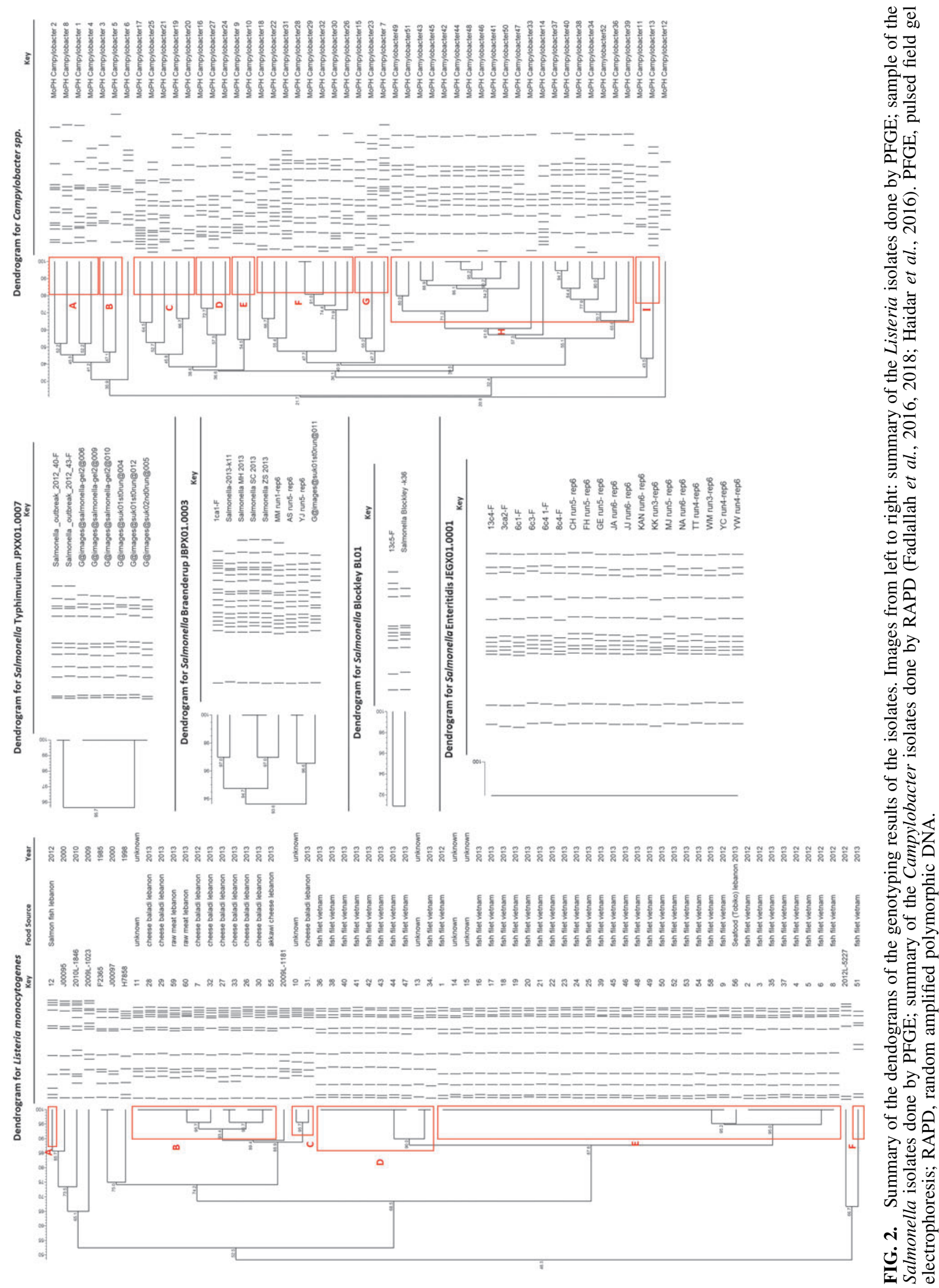


\section{Genotyping}

Regarding the Listeria isolates, PFGE analysis showed the presence of 13 different subtypes with $100 \%$ similarity that were grouped into 6 (A-E) clusters of $90 \%$ genomic similarity. The most predominant clusters were $\mathrm{E}$, which had 33 isolates (including subtypes GX6A16.0008, GX6A16.0009, and GX6A16.0010), followed by cluster B, which contained 12 isolates (including subtypes GX6A16.0000, GX6A16.0001, GX6A16.0002, and GX6A16.0003) and cluster D (including subtypes GX6A16.0006 and GX6A16.0007) with 10 isolates. Clustered subtypes were particular to the country of origin: Cluster B contained isolates from Lebanese products only (cheese and raw meat), whereas clusters D and E consisted mostly of Vietnamese fish filet (Haidar et al., 2016).

Regarding the Salmonella isolates, PFGE showed a wide range of pulsotypes. Salmonella Typhimurium isolates had 13 and 7 pulsotypes recovered from clinical and food samples, respectively. In addition, the same pulsotypes (two) of Salmonella Enteritidis were shown to be present in both food and clinical samples. There were five PFGE profiles in the clinical isolates and one in the food isolates in the Salmonella Braenderup serotype. On the other hand, Salmonella Typhi exhibited four pulsotypes in the clinical samples and three pulsotypes in the food samples (Fadlallah et al., 2016).

Eight pulsotypes of Salmonella London were identified in the clinical samples. Further, Salmonella Paratyphi A had six pulsotypes within the clinical isolates and eight pulsotypes within the food isolates. Two pulsotypes of Salmonella Blockley were isolated from the clinical and food samples. Further, there were four and one pulsotypes of Salmonella Newport isolated from food and clinical samples, respectively. Both Salmonella Paratyphi B and C had only one pulsotype in the clinical samples (Fadlallah et al., 2016).

RAPD in the Campylobacter isolates showed the presence of nine distinct clusters, namely A (8\%), B (4\%), C (10\%), D (6\%), E (4\%), F (16\%), G (6\%), H (39\%), and I (4\%). The most common RAPD type, $\mathrm{H}$, contained 20 isolates that were $55.1 \%$ genomically related; the RAPD Type $\mathrm{F}$ included 8 isolates having $47.7 \%$ genomic similarity; within this cluster, 2 isolates were genetically identical. The RAPD Type $\mathrm{C}$ had a genomic relatedness of $45.8 \%$ and contained five isolates; the RAPD Type A included four isolates, all of which had a genomic similarity of $45.5 \%$. RAPD Type D and G showed a genomic relatedness of $57.3 \%$ and $47.7 \%$, respectively, and contained three isolates. RAPD Type B, E, and I had two isolates each, showing a genomic similarity of $47.1 \%, 54.5 \%$, and $43.5 \%$, respectively (Fadlallah et al., 2018).

Figure 2 shows a summary of the genotyping results.

\section{Outbreak investigations}

During this period, PFGE was also used to link food to clinical isolates during outbreaks. During 2011, two outbreaks of Salmonella in different areas of Lebanon were identified. Clinical and suspected food samples were sent to the AUB lab for testing. The first outbreak occurred in Nabatieh during 2011. Five clinical samples were received on February 22, 2011 whereas the suspected food sample (raw meat) was received on March 4, 2011. PFGE and serotyping showed that all clinical samples and the raw meat sample were Salmonella Typhimurium with the pulsotype JPXX01. 0002. The second outbreak was identified in Mount Lebanon in September. Eight clinical samples were isolated on September 22, 2011 and two Arabic sweets were identified on October 4, 2011. The causative agent was found out to be Salmonella Enteritidis pulsotype, JEGX01.0001 (Fadlallah et al., 2016).

\section{Conclusion}

PulseNet Lebanon has been able during the past 10 years to improve surveillance and issue early warning of foodborne and waterborne outbreaks. In addition, strains of certain bacterial pathogens such as JEGX01.0001 Salmonella Enteritidis that are dominant within the region were identified. New emerging strains were able to be detected through this disease tracking network. Finally, PulseNet Lebanon was able to promote communications between laboratories and public health facilities by carrying out workshops and trainings every year in the different governorates. However, challenges remain, such as miscommunication and limited cooperation of some hospitals with the MoPH, delivery of samples in batches to the PulseNet laboratory, and turn-over of laboratory technicians.

\section{Disclosure Statement}

No competing financial interests exist.

\section{References}

Addis M, Sisay D. A review on major food borne bacterial illnesses. J Trop Dis 2015;3:176.

Clinical and Laboratory Standards Institute. Methods for Dilution Antimicrobial Susceptibility for Bacteria That Grow Aerobically; Approved Standard, 8th ed. CLSI Document M07-A8. Wayne, PA: CLSI, 2009.

Fadlallah SM, El Hajj R, El Hage J, Nasser Z, Ghosn N, Ammar W, et al. Molecular epidemiology and antimicrobial resistance of Campylobacter coli from caeca and carcass of poultry in Lebanon. Acta Sci Microbiol J 2018;1.4:63-70.

Fadlallah SM, Shehab M, Cheaito K, Selah M, Ghosn N, Ammar W, et al. Molecular epidemiology and antimicrobial resistance of Salmonella species from clinical specimens and food items in Lebanon. J Infect Dev Ctries 2016; 10:712-717.

Fernández H, Pisón V. Isolation of thermotolerant species of Campylobacter from commercial chicken livers. Int $\mathbf{J}$ Food Microbiol 1996;29:75-80.

Ghosn N, Nasredine A, Baddour YM, Coulombier D, Nasserdine S. Electronic surveillance of outbreaks in Lebanon. BMC Proc 2008;2(Suppl 3):S2.

Haidar N, Kissoyan, KAB, Fadlalah SM, El Hajj R, Selah M, Ghosn $\mathrm{N}$, et al. Genotypic and virulence characteristics of Listeria monocytogenes recovered from food items in Lebanon. J Infect Dev Ctries 2016;10:712-717.

Pezzotti G, Serafin A, Luzzi I, et al. Occurrence and resistance to antibiotics of Campylobacter jejuni and Campylobacter coli in animals and meat in northeastern Italy. Int $\mathbf{J}$ Food Microbiol 2003;82:281-287.

PulseNet International. Standard Operating Procedure for Pulsenet PFGE of Listeria monocytogenes. 2013. Available at: www.pulsenetinternational.org/assets/PulseNet/uploads/pfge/ PNL04_ListeriaPFGEProtocol.pdf, accessed August 20, 2018.

Ribot EM, Fair MA, Gautom R, Cameron DN, Hunter SB, Swaminathan B, et al. Standardization of pulsed-field gel 
electrophoresis protocols for the subtyping of Escherichia coli O157:H7, Salmonella, Shigella for Pulsenet. Foodborne Pathog Dis 2006;3:59-67.

Talhouk RS, el-Dana RA, Araj GF, et al. Prevalence, antimicrobial susceptibility and molecular characterization of Campylobacter isolates recovered from humans and poultry in Lebanon. J Med Liban 1998;46:310-316.

Wolfram T. Most Common Foodborne Pathogens. Academy of Nutrition and Dietetics. Chicago, 2017. Available at: www .eatright.org/homefoodsafety/safety-tips/food-poisoning/mostcommon-foodborne-pathogens, accessed August 25, 2018.

World Health Organization. National Health Statistics Report in Lebanon. Geneva, 2012. Available at: www.igsps.usj.edu .lb/docs/recherche/recueil12en.pdf, accessed August 18, 2018

World Health Organization. Advancing the Right to Health: The Vital Role of Law. Geneva, 2017. Available at: http://apps .who.int/iris/bitstream/handle/10665/252815/9789241511 384-eng.pdf; jsessionid $=$ 09C2C9798C28ED3BE652D120658 9A11D? sequence =1, accessed August 18, 2018.

Zhao C, Ge B, De Villena J, et al. Prevalence of Campylobacter spp., Escherichia coli, and Salmonella serovars in retail chicken, turkey, pork, and beef from the Greater Washington, D.C., area. Appl Environ Microbiol 2001;67:5431-5436.

Address correspondence to: Ghassan M. Matar, PhD Department of Experimental Pathology, Immunology and Microbiology

Faculty of Medicine American University of Beirut

Beirut 10017

Lebanon

E-mail: gmatar@aub.edu.lb 